\title{
Combining Ability for Yield and Yield Components through Diallel Analysis in Okra [Abelmoschus esculentus (L.) Moench]
}

\author{
M. Amaranatha Reddy ${ }^{1^{*}}$ and O. Sridevi ${ }^{2}$ \\ ${ }^{1}$ Department of Genetics and Plant Breeding, College of Horticulture, Anantharajupeta, YSR \\ Kadapa, A.P - 516105, India \\ ${ }^{2}$ Department of Genetics and Plant Breeding, College of Agriculture, University of \\ Agricultural Sciences, Dharwad - 580 005, Karnataka, India \\ *Corresponding author
}

\begin{tabular}{|c|}
\hline Keywords \\
\hline $\begin{array}{l}\text { Combining ability, } \\
\text { Gene action, } \\
\text { Diallel, Fruit yield, } \\
\text { Okra }\end{array}$ \\
\hline Article Info \\
\hline $\begin{array}{l}\text { Accepted: } \\
\text { 10 February } 2018 \\
\text { Available Online: } \\
10 \text { March } 2018\end{array}$ \\
\hline
\end{tabular}

\section{Introduction}

Okra (Abelmoschus esculentus (L.) Moench), one of the important vegetable crops of India, belongs to family Malvaceae and the genus Abelmoschus. It is an economically important vegetable crop grown in tropical and subtropical parts of the world. It is native of tropical Africa. It is called lady's finger in England, gumbo in the United States of America, guino-gombo in Spanish, guibeiro in Portuguese and bhendi in India. It is often cross pollinated crop and thus heterosis can be exploited in it. Breeding method for the improvement of a crop depends primarily on the nature and magnitude of gene action involved in the expression of quantitative and qualitative traits. Combining ability analysis helps in the identification of parents with high general combining ability (GCA) effects and cross combinations with high specific combining ability (SCA) effects. Additive and non additive gene actions in the parents estimated through combining ability analysis 
may be useful in determining the possibility for commercial exploitation of heterosis and isolation of purelines among the progenies of the heterotic $F_{1}$. The present study was conducted to obtain the information on combining ability of 8 varieties of Okra (Abelmoschus esculentus L.) for fruit yield and its components.

\section{Materials and Methods}

Eight parents viz., Arka Anamika, Arka Abhay, DBh-30, DBh-37, DBh-39, DBh-43, DBh-47 and DBh-55 selected and were crossed in full diallel fashion to analyse the combining ability and heterosis for yield and yield component traits. Fifty six hybrids, eight parents along with four popular hybrids (Syngenta 152, Mahyco No. 10, Mahyco No. 55, and Mahyco No. 64) were evaluated in three replications of Randomized Block Design during rabi season of 2011-2012.

Observations were recorded on five competitive plants excluding border plants in each replication for days to 50 per cent flowering, plant height, number of branches, inter-nodal length, fruit length, fruit diameter, number of fruits per plant, fruit weight, fruit yield per plant and fruit yield per hectare. As the data was obtained from chosen set of parents along with direct and reciprocal crosses, method 1 and model 1 of Griffing (1956) was employed for the analysis.

\section{Results and Discussion}

The variance due to treatments was found highly significant for all the characters studied. The parents and hybrids exhibited highly significant variation for all the characters studied. It indicates significant difference among parents and hybrids. Parents Vs hybrids exhibited significant variation for days to $50 \%$ flowering, plant height, number of branches days, inter-nodal length, number of fruits, fruit weight, average fruit yield per plant and yield per hectare and non-significant variation for fruit length and fruit diameter. Variance due to $F_{1}$ 's and reciprocal was found significant for all characters studied. Variance due to $\mathrm{F}_{1}$ 's $\mathrm{v} / \mathrm{s}$ reciprocal interaction was highly significant for days to $50 \%$ flowering, number of branches per plant, inter-nodal length, number of fruits, average fruit yield per plant and non-significant for plant height, fruit length, fruit diameter, fruit weight and fruit yield per hectare (Table 1).

The mean sum of squares due to SCA, GCA, reciprocals were found highly significant for days to 50 per cent flowering, plant height, number of branches, inter-nodal length, fruit length, fruit diameter, number of fruits per plant, fruit weight, fruit yield per plant and fruit yield per hectare. The estimates of SCA variance were high for all characters than GCA variance except for fruit length. The proportion of variance due to GCA/SCA was found to be less than one for all the characters except for fruit length.

The estimates of SCA variances were high for all the characters viz., days to 50 per cent flowering, plant height, number of branches per plant, inter nodal length, fruit diameter, number of fruits per plant, fruit weight, fruit yield per plant and fruit yield per hectare indicating predominance of non-additive gene action in determining these traits. Dhankar and Dhankar (2002), Dahake and Bangar (2006) and Sanjay Singh et al., (2006) reported a major role of non-additive gene action on days to 50 per cent flowering. The predominance of SCA variance for fruit weight and fruit diameter has been reported by Sharma and Mahajan (1978), Vijay and Manohar (1986), Chaudhary et al., (1991), Dahake and Bangar (2006) and Sharma and Mahajan (1978). The predominance of SCA variance for fruit weight and fruit diameter has been reported by Sharma and Mahajan (1978), Vijay and 
Manohar (1986), Chaudhary et al., (1991) and Dahake and Bangar (2006). Non-additive gene action as a predominant factor in determining the number of branches per plant and number of fruits per plant was reported by Sharma and Mahajan (1978), Shukla et al., (1989), Senthil Kumar et al., (2006) and Ahmed et al., (1997). Poshiya and Shukla (1986), Vijay and Manohar (1986) and Sanjay Singh et al., (2006) reported that predominance of nonadditve gene action for fruit yield per plant.

The proportion of variance due to GCA/SCA was found to be more than one for fruit length which was determined by additive gene action (Table 2). Yield parameters like number of fruits per plant and average fruit weight predominantly controlled by non-additive gene action and hence these traits can be exploited through heterosis breeding. The predominance of GCA variance for fruit length, indicates additive gene action in expression of this character. Vijay and Manohar (1986), Sivagamasundari et al., (1992) and Wankhade et al., (1995) obtained similar results.

The estimates of gca effects revealed that Arka anamika and DBh-30 for lesser days to fifty per cent flowering, DBh-37 and 39 for higher plant height, Arka abhay and DBh-47 for more number of branches per plant, DBh47 and Arka abhay for lesser inter nodal length, Arka anamika and Arka abhay for higher fruit length, DBh-43 and 39 for reduced fruit diameter, Arka anamika and Arka abhay for more number of fruits per plant, Arka anmika and DBh-30 for higher fruit weight, Arka anamika and Arka abhay for higher yield per plant and fruit yield per hectare.

The parent Arka anamika showed higher combining ability for six characters viz., days to 50 per cent flowering, fruit length, number of fruits per plant, fruit weight, fruit yield per plant and fruit yield per hectare followed by the parent Arka abhay (Table 3). $F_{2}$ and later segregating population from cross combinations involving parents with high gca effects can be used for participating selection.

In case of days to 50 per cent flowering, Arka Anamika (-1.255) recorded highest significant negative gca effect and The highest significant negative sca effect was observed in DBh-55 x Arka Anamika (-2.167) cross followed by DBh-43 x Arka Abhay (-2.00) and Arka Abhay $x$ DBh-37 (-1.682). The sca effect ranged from -2.167 (DBh-55 x Arka Anamika) to 1.833 (DBh-37 x Arka Abhay). 17 hybrids recorded significant negative sca effect which is considered to be desirable since, earliness is desirable.

The sca effects ranged from -26.183 to 15.1 for the plant height, -0.833 to 0.667 for number of branches, -0.7 to 0.733 for internodal length, -0.617 to 0.983 for fruit length, 8.333 to 11.667 for fruit diameter, -1.458 to 2.167 for average fruit weight and -2.583 to 2.627 for fruit yield per hectare.

In case of fruit yield per plant, the highest gca effect recorded in Arka Anamika (1.726) followed by Arka Abhay (0.457). The sca effect ranged from -2.583 (DBh-37 x Arka Abhay) to 2.627 (Arka Anamika x DBh-43). The highest significant positive sca effect was observed in the cross Arka Anamika x DBh43 (2.627) followed by DBh-47 x Arka Anamika (2.032) and DBh-47 x DBh-30 (1.607). Among 56 hybrids tested, 16 hybrids recorded significant positive sca effects (Table 4).

Based on the total score values it is observed that among the parents Arka Anmika had higher gca scores and is a good combiner for days to 50 per cent flowering, fruit length, number of fruits per plant, fruit weight, fruit yield per plant and fruit yield per hectare, followed by Arka Abhay and DBh-43. 
Table.1 Analysis of variance (mean sum of square) for fruit yield and its component traits in okra

\begin{tabular}{|c|c|c|c|c|c|c|c|c|c|c|c|}
\hline Character & d.f. & $\begin{array}{c}\text { Days to } \\
50 \% \\
\text { flowering }\end{array}$ & $\begin{array}{l}\text { Plant height } \\
\text { (cm) }\end{array}$ & $\begin{array}{l}\text { Number of } \\
\text { branches } \\
\text { per plant }\end{array}$ & $\begin{array}{l}\text { Inter } \\
\text { nodal } \\
\text { length } \\
(\mathrm{cm})\end{array}$ & $\begin{array}{c}\text { Fruit } \\
\text { length } \\
\text { (cm) }\end{array}$ & $\begin{array}{c}\text { Fruit } \\
\text { Diameter } \\
(\mathbf{c m})\end{array}$ & $\begin{array}{l}\text { Number } \\
\text { of fruits } \\
\text { per plant }\end{array}$ & $\begin{array}{l}\text { Average } \\
\text { fruit } \\
\text { weight (g) }\end{array}$ & $\begin{array}{l}\text { Fruit yield per } \\
\text { plant (g) }\end{array}$ & $\begin{array}{c}\text { Fruit } \\
\text { yield per } \\
\text { hectare } \\
(\mathrm{T} / \mathrm{ha})\end{array}$ \\
\hline Replicates & 2 & 1.943 & 7.06 & 0.021 & 0.11 & 0.46 & 63.021 & 0.328 & 0.161 & 63.453 & 0.715 \\
\hline Treatments & 63 & $9.680^{* *}$ & $527.220 * *$ & $0.735^{* *}$ & $1.037 * *$ & $1.406^{* *}$ & $134.053 * *$ & $15.497 * *$ & $3.603 * *$ & $4030.338 * *$ & $10.314 * *$ \\
\hline Parents & 7 & $3.310^{* *}$ & $383.501 * *$ & $1.048^{* *}$ & $0.911^{* *}$ & $2.486^{* * *}$ & 190.476 ** & $23.708 * *$ & $2.381 * *$ & $4714.736 * *$ & 14.480 ** \\
\hline Hybrids & 55 & $9.787 * *$ & $529.491 * *$ & $0.696^{* *}$ & $0.927 * *$ & $1.281^{* *}$ & $128.820 * *$ & $14.018 * *$ & $3.678 * *$ & $3773.176 * *$ & $8.951 * *$ \\
\hline $\begin{array}{l}\text { Parent Vs. } \\
\text { Hybrids }\end{array}$ & 1 & $48.382 * *$ & $1408.352 * *$ & $0.715^{*}$ & $7.955^{* *}$ & 0.679 & 26.86 & $39.360 * *$ & $8.048 * *$ & $13383.510^{* * *}$ & $56.146^{* *}$ \\
\hline $\mathrm{F}_{1}$ 's & 27 & $11.247 * *$ & $606.506^{* *}$ & $0.619 * *$ & $0.902 * *$ & $1.116^{* * *}$ & $154.630 * *$ & $14.827 * *$ & $4.802 * *$ & $4475.661 * *$ & $10.902 * *$ \\
\hline Reciprocals & 27 & $8.354 * *$ & $471.788 * *$ & $0.750^{* * *}$ & 0.863 ** & $1.494^{* * *}$ & $107.231 * *$ & $13.321 * *$ & $2.224 * *$ & $3122.593 * *$ & $7.280^{* *}$ \\
\hline $\begin{array}{l}\mathrm{F}_{1} \text { Vs } \\
\text { Reciprocals }\end{array}$ & 1 & $9.054 * *$ & 8.061 & $1.339 * *$ & $3.343 * *$ & 0.005 & 14.881 & $11.006 * *$ & 12.595 & $2371.810 * *$ & 1.414 \\
\hline Error & 126 & 0.959 & 35.549 & 0.148 & 0.089 & 0.177 & 40.799 & 1.566 & 1.050 & 89.282 & 0.958 \\
\hline Total & 191 & 3.846 & 197.425 & 0.34 & 0.402 & 0.585 & 71.79 & 6.148 & 1.883 & 1388.941 & 4.042 \\
\hline
\end{tabular}

\footnotetext{
* - Significant at $5 \% \quad * *$ - Significant at $1 \%$
}

Table.2 ANOVA for combining ability for Fruit yield and yield component traits in okra

\begin{tabular}{|c|c|c|c|c|c|c|c|c|c|c|}
\hline Source & GCA & SCA & Reciprocal & Error & & \multicolumn{5}{|c|}{ Fixed effects } \\
\hline df & 7 & 28 & 28 & 126 & $\sigma \gamma^{\prime \prime}$ & $\sigma \sigma^{\prime \prime}$ & $\begin{array}{c}\mathrm{GCA} / \mathrm{S} \\
\mathrm{CA}\end{array}$ & $\sigma \iota \chi \varepsilon \rho "$ & $\sigma^{2} \alpha$ & $\sigma \Delta^{\prime \prime}$ \\
\hline Days to $50 \%$ flowering & $10.698 * *$ & $2.413^{* *}$ & $2.173 * *$ & 0.32 & 0.649 & 2.093 & 0.31 & 0.927 & 1.297 & 2.093 \\
\hline Number of branches & $0.390 * *$ & $0.261 * *$ & $0.192 * *$ & 0.049 & 0.021 & 0.212 & 0.099 & 0.072 & 0.043 & 0.212 \\
\hline Inter nodal length $(\mathrm{cm})$ & $0.453^{* *}$ & $0.326^{* *}$ & $0.338 * *$ & 0.03 & 0.026 & 0.297 & 0.088 & 0.154 & 0.053 & 0.297 \\
\hline Number of fruits & $19.819 * *$ & $3.245^{* *}$ & $3.423^{* *}$ & 0.522 & 1.206 & 2.723 & 0.443 & 1.45 & 2.412 & 2.723 \\
\hline Average fruit weight (g) & $0.613^{* *}$ & $1.207 * *$ & $1.341 * *$ & 0.35 & 0.016 & 0.86 & 0.019 & 0.496 & 0.033 & 0.857 \\
\hline Fruit yield per plant (g) & $\begin{array}{c}4682.361 * \\
*\end{array}$ & $865.913^{* *}$ & $986.251 * *$ & 29.761 & 290.788 & 836.15 & 0.348 & 478.245 & $\begin{array}{c}581.57 \\
5\end{array}$ & 836.15 \\
\hline Fruit yield per hectare (T) & $12.593 * *$ & $2.532 * *$ & $2.056^{* * *}$ & 0.319 & 0.767 & 2.212 & 0.347 & 0.868 & 1.534 & 2.212 \\
\hline
\end{tabular}

* - Significant at $5 \%$

** - Significant at $1 \%$

Table.3 GCA effects of parents for yield and yield component traits in okra

\begin{tabular}{|c|c|c|c|c|c|c|c|c|c|c|c|}
\hline Parents & $\begin{array}{c}\text { Days to } 50 \% \\
\text { flowering }\end{array}$ & $\begin{array}{c}\text { Plant } \\
\text { height }(\mathbf{c m})\end{array}$ & $\begin{array}{l}\text { Number of } \\
\text { branches } \\
\text { per plant }\end{array}$ & $\begin{array}{c}\text { Inter } \\
\text { nodal } \\
\text { length } \\
(\mathrm{cm})\end{array}$ & $\begin{array}{c}\text { Fruit } \\
\text { length } \\
\text { (cm) }\end{array}$ & $\begin{array}{c}\text { Fruit } \\
\text { diameter } \\
(\mathbf{c m})\end{array}$ & $\begin{array}{c}\text { Number of } \\
\text { fruits per } \\
\text { plant }\end{array}$ & $\begin{array}{c}\text { Average } \\
\text { fruit } \\
\text { weight (g) }\end{array}$ & $\begin{array}{c}\text { Fruit yield } \\
\text { per plant } \\
\text { (g) }\end{array}$ & $\begin{array}{l}\text { Fruit yield } \\
\text { per hectare } \\
\text { (T) }\end{array}$ & $\begin{array}{l}\text { Total } \\
\text { Score }\end{array}$ \\
\hline Arka Abhay & $-0.339^{*}$ & $-10.941 * *$ & $0.255^{* *}$ & -0.061 & $0.330^{* *}$ & $-2.448 * *$ & $1.031^{* *}$ & -0.208 & $8.854 * *$ & $0.457 * *$ & 4 \\
\hline $\begin{array}{l}\text { Arka } \\
\text { Anamika }\end{array}$ & $-1.255 * *$ & $-2.070 *$ & 0.047 & -0.014 & $0.849 * *$ & -0.156 & $1.719 * *$ & $0.375 * *$ & $34.328 * *$ & $1.726^{* *}$ & 5 \\
\hline DBh-30 & $-0.714 * *$ & $-5.030 * *$ & $-0.161 * *$ & 0.076 & $-0.309 * *$ & -1.198 & 0.052 & 0.021 & -1.552 & -0.095 & -2 \\
\hline DBh-37 & $0.932 * *$ & 10.849 ** & -0.036 & $0.174 * *$ & -0.039 & -0.573 & -0.052 & 0.042 & $-5.104 * *$ & $-0.310^{*}$ & -3 \\
\hline DBh-39 & $1.224 * *$ & $6.605^{* *}$ & -0.078 & $0.095^{*}$ & $-0.272^{* *}$ & $2.135^{*}$ & $-0.365^{*}$ & -0.104 & $-4.468 * *$ & -0.231 & -4 \\
\hline DBh-43 & $0.266^{*}$ & $3.118^{* * *}$ & 0.089 & $0.126^{* *}$ & 0.018 & $3.594 * *$ & $0.469 * *$ & 0.083 & $4.585^{* *}$ & $0.416^{* *}$ & 3 \\
\hline DBh-47 & -0.193 & -1.441 & $0.109 *$ & $-0.364 * *$ & $-0.278 * *$ & -0.573 & $-1.094 * *$ & 0.042 & $-13.273 * *$ & $-0.804 * *$ & -2 \\
\hline DBh-55 & 0.078 & -1.091 & $-0.224 * *$ & -0.032 & $-0.299 * *$ & -0.781 & $-1.760 * *$ & -0.250 & $-23.370 * *$ & $-1.159 * *$ & -5 \\
\hline
\end{tabular}

\footnotetext{
* - Significant at 5\% ** - Significant at $1 \%$
} 
Table.4 SCA effects of hybrids for different yield and yield component traits in okra

\begin{tabular}{|c|c|c|c|c|c|c|c|c|c|c|c|}
\hline \multirow{2}{*}{\multicolumn{2}{|c|}{ Arka Abhav x Arka Anamika }} & $\begin{array}{c}\text { Plant } \\
\text { height }(\mathbf{c m})\end{array}$ & $\begin{array}{c}\text { Number of } \\
\text { branches } \\
\text { Per plant }\end{array}$ & $\begin{array}{l}\text { Inter nodal } \\
\text { length }(\mathrm{cm})\end{array}$ & $\begin{array}{c}\text { Fruit } \\
\text { length } \\
(\mathrm{cm})\end{array}$ & $\begin{array}{c}\text { Fruit } \\
\text { Diameter } \\
(\mathbf{c m})\end{array}$ & $\begin{array}{c}\text { Number } \\
\text { of fruits } \\
\text { per plant }\end{array}$ & $\begin{array}{c}\text { Average } \\
\text { fruit } \\
\text { weight (g) }\end{array}$ & $\begin{array}{l}\text { Fruit yield } \\
\text { per plant (g) }\end{array}$ & $\begin{array}{c}\text { Fruit yield } \\
\text { per hectare } \\
\text { (T) }\end{array}$ & $\begin{array}{l}\text { Total } \\
\text { Score }\end{array}$ \\
\hline & -0.16 & -4.25 & 0.03 & $0.65 * *$ & -0.18 & $-6.71 * *$ & 0.17 & 0.54 & $34.56^{* *}$ & $-1.01^{* *}$ & -2 \\
\hline \multicolumn{2}{|l|}{ Arka Abhay x DBh-30 } & $-7.14 * *$ & $0.57 * *$ & $0.51^{* *}$ & 0.27 & -0.67 & $1.5^{* *}$ & $-1.10^{*}$ & $-7.73 *$ & 0.46 & -3 \\
\hline \multicolumn{2}{|l|}{ Arka Abhay x DBh-37 } & 1.87 & $-0.38^{*}$ & $-0.38 * *$ & 0.15 & 3.69 & $0.94 *$ & 0.70 & $-44.09 *$ & $1.43 * *$ & 2 \\
\hline \multicolumn{2}{|l|}{ Arka Abhay x DBh-39 } & -1.29 & -0.00 & $-0.3 * *$ & -0.11 & 4.32 & $0.92 *$ & 0.68 & $-12.29 * *$ & $0.75^{*}$ & 2 \\
\hline Arka Abhay x DBh-43 & -0.8 & 4.02 & $0.66 * *$ & $0.4 * *$ & $0.31 *$ & 1.19 & $-1.24 *$ & 0.50 & $-7.84 *$ & -0.07 & 0 \\
\hline Arka Abhay x DBh-47 & 0.4 & 1.01 & -0.02 & -0.06 & -0.18 & -2.96 & -0.51 & -0.45 & -1.59 & $-1.00^{* *}$ & -1 \\
\hline Arka Abhay x DBh-55 & 0.0 & 0.93 & -0.02 & $0.43 * *$ & -0.21 & -2.76 & $-1.01^{*}$ & -0.50 & $-10.06^{* *}$ & -0.56 & -3 \\
\hline Arka Anamika x Arka Abhay & -0.1 & 0.66 & $0.33^{*}$ & 0.13 & -0.28 & -1.66 & $2.33 * *$ & -0.16 & $-12.51 * *$ & $1.38 * *$ & 2 \\
\hline Arka Anamika x DBh-30 & 0.1 & -1.59 & -0.21 & 0.06 & $-0.60 * *$ & $-6.30 *$ & $-1.84 * *$ & $-1.18 * *$ & $-13.87 * *$ & $-1.71 * *$ & -6 \\
\hline Arka Anamika x DBh-37 & -0.9 & $14.65 * *$ & -0.17 & $0.51 * *$ & -0.03 & $6.40 * *$ & 0.76 & -0.04 & $13.20 * *$ & $0.74 *$ & 4 \\
\hline Arka Anamika x DBh-39 & -1.22 & 2.69 & 0.20 & 0.11 & 0.03 & 2.03 & 0.073 & -0.06 & -1.32 & 0.35 & 1 \\
\hline Arka Anamika x DBh-43 & -0.4 & $10.35 * *$ & $0.37 *$ & -0.03 & $0.31 *$ & 3.90 & $3.07 * *$ & 0.25 & $26.11 * *$ & $2.62 * *$ & 6 \\
\hline Arka Anamika x DBh-47 & 0.1 & -3.98 & $-0.48 * *$ & $0.31 * *$ & $0.39 *$ & -0.26 & -0.86 & $1.45 * *$ & $52.85^{* *}$ & $0.98^{* *}$ & 2 \\
\hline Arka Anamika x DBh-55 & -0.9 & -1.97 & $0.34 *$ & $0.73 * *$ & 0.26 & -1.71 & 0.46 & -0.08 & $-13.79 * *$ & 0.28 & 0 \\
\hline DBh-30 x Arka Abhay & 0.3 & -1.28 & $0.66 * *$ & 0.04 & 0.11 & -3.33 & 1.00 & -0.83 & 3.02 & -0.00 & 1 \\
\hline DBh-30 x Arka Anamika & -0.8 & -0.06 & $0.33^{*}$ & $-0.28 *$ & -0.21 & $-6.66^{*}$ & 0.00 & 0.00 & $-31.31 * *$ & -0.77 & 1 \\
\hline DBh-30 x DBh-37 & 0.1 & -1.58 & $-0.29 *$ & $-0.4 * *$ & -0.01 & 4.11 & $-1.57 * *$ & -0.02 & $-8.88^{*}$ & 0.012 & -2 \\
\hline DBh-30 x DBh-39 & -1.26 & $6.22 * *$ & 0.07 & 0.00 & 0.09 & $-6.92 * *$ & 0.073 & 0.45 & $-13.26 * *$ & 0.34 & 0 \\
\hline DBh-30 x DBh-43 & -1.47 & 0.21 & 0.07 & $-0.31^{* *}$ & $-0.37 *$ & -0.05 & 0.24 & -0.06 & -3.09 & -0.23 & 1 \\
\hline DBh-30 x DBh-47 & -0.8 & 0.60 & 0.22 & $0.36 * *$ & $0.31 *$ & 2.44 & -0.19 & -0.02 & $35.83 * *$ & 0.41 & 2 \\
\hline DBh-30 x DBh-55 & -0.7 & $11.58 * *$ & $-0.44 * *$ & $0.71 * *$ & $0.40 *$ & $5.99 *$ & $0.96 *$ & 0.77 & -3.01 & $0.94 *$ & 4 \\
\hline DBh-37 x Arka Abhay & 1.83 & $-26.18 * *$ & $0.5^{* *}$ & $0.54 * *$ & -0.03 & -5.00 & $-3.33 * *$ & 0.33 & $30.10^{* *}$ & $-2.58 * *$ & -3 \\
\hline DBh-37 x Arka Anamika & -1.33 & -1.53 & $0.5^{* *}$ & 0.03 & $-0.46^{*}$ & 0.00 & 0.16 & $1.16^{* * *}$ & $12.41^{* *}$ & 0.72 & 3 \\
\hline DBh-37 x DBh-30 & -0.6 & $-11.73 *$ & -0.16 & -0.24 & $-0.4 *$ & 0.00 & 0.16 & -0.5 & -6.99 & $-0.95 *$ & -3 \\
\hline DBh-37 x DBh-39 & 0.7 & $-6.65^{* *}$ & $0.28 *$ & $-0.35 * *$ & 0.12 & 4.11 & $-0.9^{*}$ & $-1.22 * *$ & $-15.76^{* *}$ & $-0.89 *$ & -4 \\
\hline DBh-37 x DBh-43 & 1.21 & 1.26 & -0.04 & -0.13 & -0.04 & $-7.34 * *$ & -0.32 & 0.08 & 2.57 & -0.56 & -2 \\
\hline DBh-37 x DBh-47 & 0.5 & 2.62 & -0.06 & $0.23^{*}$ & 0.01 & 0.15 & 0.40 & -0.04 & $22.83 * *$ & 0.01 & 0 \\
\hline & & & & & & & & & Contd. & ... & \\
\hline Crosses & $\begin{array}{c}\text { Days to } \\
\mathbf{5 0 \%} \\
\text { flowering }\end{array}$ & $\begin{array}{c}\text { Plant } \\
\text { height }(\mathbf{c m})\end{array}$ & $\begin{array}{l}\text { Number of } \\
\text { branches } \\
\text { Per plant }\end{array}$ & $\begin{array}{l}\text { Inter nodal } \\
\text { length }(\mathbf{c m})\end{array}$ & $\begin{array}{l}\text { Fruit } \\
\text { length } \\
(\mathbf{c m})\end{array}$ & $\begin{array}{l}\text { Fruit } \\
\text { Diameter } \\
\text { (cm) }\end{array}$ & $\begin{array}{l}\text { Number } \\
\text { of fruits } \\
\text { per plant }\end{array}$ & $\begin{array}{c}\text { Average } \\
\text { fruit } \\
\text { weight }(g)\end{array}$ & $\begin{array}{c}\text { Fruit yield } \\
\text { per plant } \\
\text { (g) }\end{array}$ & $\begin{array}{l}\text { Fruit yield } \\
\text { per hectare } \\
\text { (T) }\end{array}$ & $\begin{array}{l}\text { Total } \\
\text { Score }\end{array}$ \\
\hline DBh-37 x DBh-55 & -0.266 & 4.143 & -0.068 & $0.25^{*}$ & -0.166 & -1.302 & $1.406^{* *}$ & -0.25 & 5.237 & 0.233 & 0 \\
\hline DBh-39 x Arka Abhay & 0.167 & $-6.6^{*}$ & 0.167 & 0.207 & -0.2 .00 & -1.667 & -1.00 & -0.5 .00 & $10.874 * *$ & -0.382 & 0 \\
\hline DBh-39 x Arka Anamika & $-1.00 *$ & $-12.4 * *$ & -0.167 & $0.343 * *$ & $-0.567 * *$ & $-8.333 * *$ & 0.500 & 0.667 & 6.538 & -0.073 & -3 \\
\hline DBh-39 x DBh-30 & -0.500 & -1.167 & -0.167 & $0.37 * *$ & 0.100 & 1.667 & -0.500 & -0.167 & $9.515^{*}$ & -0.733 & 0 \\
\hline DBh-39 x DBh-37 & 0.167 & -1.833 & -0.167 & $-0.278 *$ & 0.00 & -3.333 & -1.00 & -0.167 & $-20.408 * *$ & -0.472 & 0 \\
\hline DBh-39 x DBh-43 & $1.422 * *$ & -2.755 & 0.161 & $0.717 * *$ & -0.099 & 1.615 & -0.177 & -0.271 & $39.858 * *$ & -0.076 & -1 \\
\hline DBh-39 x DBh-47 & -0.286 & $6.97 * *$ & -0.026 & $0.267^{*}$ & 0.08 & 4.115 & $1.552 * *$ & 0.104 & $34.282 * *$ & 0.269 & 2 \\
\hline DBh-39 x DBh-55 & 0.276 & 2.586 & -0.193 & $0.283^{*}$ & 0.084 & -0.677 & 0.219 & -0.104 & $21.145 * *$ & $1.091 * *$ & 1 \\
\hline DBh-43 x Arka Abhay & $-2.00 * *$ & -3.433 & 0.00 & $0.343 * *$ & $-0.617 * *$ & 0.00 & -1.00 & 0.500 & -4.131 & -0.733 & -1 \\
\hline DBh-43 x Arka Anamika & $-1.500^{* *}$ & $15.1^{* *}$ & $-0.833^{* *}$ & 0.028 & 0.200 & 1.667 & 1.00 & $2.167 * *$ & $50 * *$ & $1.448 * *$ & 4 \\
\hline DBh-43 x DBh-30 & $1.667 * *$ & -2.667 & 0.00 & $0.355^{* * *}$ & 0.317 & $-6.667 *$ & 0.167 & -0.500 & 1.585 & -0.173 & -3 \\
\hline DBh-43 x DBh-37 & 0.333 & $5.60^{*}$ & 0.00 & 0.107 & -0.217 & -3.333 & 0.50 & -0.667 & -4.571 & 0.132 & 1 \\
\hline DBh-43 x DBh-39 & -0.500 & $9.00 * *$ & 0.167 & -0.097 & -0.133 & 5.00 & $2.333^{* *}$ & 0.833 & $-9.611 *$ & $1.402 * *$ & 2 \\
\hline DBh-43 x DBh-47 & 0.339 & $-5.976^{* *}$ & -0.193 & $0.35^{* *}$ & -0.226 & -2.344 & 0.552 & -0.083 & 6.77 & 0.544 & -2 \\
\hline DBh-43 x DBh-55 & -0.099 & $-5.459^{*}$ & 0.141 & $-0.3^{* *}$ & -0.122 & $-5.469^{*}$ & $-1.448^{* *}$ & $-1.458^{* * *}$ & $-10.383^{* *}$ & $-1.052^{* *}$ & -5 \\
\hline DBh-47 x Arka Abhay & -0.500 & 0.00 & $0.333^{*}$ & -0.101 & -0.017 & -5.00 & 0.167 & -0.500 & $-18.443 * *$ & 0.005 & 0 \\
\hline DBh-47 x Arka Anamika & -0.667 & $6.00^{*}$ & $-0.333^{*}$ & $0.434 * *$ & $0.983 * *$ & 3.333 & 0.50 & $2.00 * *$ & $11.306 * *$ & $2.032 * *$ & 3 \\
\hline DBh-47 x DBh-30 & -0.500 & 4.967 & 0.167 & $0.395 * *$ & $0.383 *$ & 1.667 & $2.5 * *$ & 0.500 & 2.174 & $1.607 * *$ & 2 \\
\hline DBh-47 x DBh-37 & 0.167 & $8.267 * *$ & 0.00 & 0.164 & $0.683 * *$ & 0.00 & 1.00 & 0.167 & -1.294 & $1.103^{*}$ & 3 \\
\hline DBh-47 x DBh-39 & $1.00 *$ & $10.833^{* *}$ & 0.00 & 0.076 & 0.317 & $6.667^{*}$ & $2.167^{* *}$ & $1.167 * *$ & $25.017 * *$ & $0.903 *$ & 5 \\
\hline DBh-47 x DBh-43 & $1.00^{*}$ & $5.933^{*}$ & 0.00 & $-0.539 * *$ & 0.200 & 5.00 & -0.333 & -0.167 & 1.342 & 0.748 & 1 \\
\hline DBh-47 x DBh-55 & -0.474 & -0.434 & 0.12 & $-0.7 * *$ & 0.141 & $5.365^{*}$ & $-1.615^{* *}$ & -0.75 & $-23.053^{* *}$ & $1.407^{* * *}$ & 1 \\
\hline DBh-55 x Arka Abhay & $-1.00^{*}$ & 0.733 & 0.00 & $-0.366 * *$ & -0.067 & -5.00 & -0.333 & 0.833 & $-8.152^{*}$ & -0.392 & 1 \\
\hline DBh-55 x Arka Anamika & $-2.167 * *$ & $5.567^{*}$ & 0.167 & -0.147 & 0.00 & 1.667 & $1.167^{*}$ & 0.833 & 7.644 & -0.752 & 3 \\
\hline DBh-55 x DBh-30 & 0.50 & 0.567 & 0.167 & $0.68 * *$ & -0.083 & 1.667 & 0.333 & 0.00 & $22.445^{* *}$ & -0.168 & 0 \\
\hline DBh-55 x DBh-37 & -0.667 & 2.733 & $0.333^{*}$ & $0.249^{*}$ & 0.35 & $11.667^{* *}$ & 1.00 & $-1.000^{*}$ & 6.115 & 0.117 & 0 \\
\hline DBh-55 x DBh-39 & $-1.5^{* *}$ & 2.533 & $0.5^{* *}$ & 0.161 & 0.100 & 5.00 & $-1.833^{* *}$ & 0.667 & 7.738 & $0.937 *$ & 2 \\
\hline DBh-55 x DBh-43 & $0.833^{*}$ & -0.40 & 0.00 & $-0.42 *$ & 0.117 & 1.667 & 0.667 & 0.500 & $-28.657 * *$ & -0.665 & -1 \\
\hline DBh-55 x DBh-47 & $1.00 *$ & -3.067 & 0.00 & -0.03 & $-0.417 *$ & -5.00 & $-1.167^{*}$ & 0.500 & $26.617 * *$ & $-1.11^{* *}$ & -3 \\
\hline
\end{tabular}

* - Significant at $5 \% \quad * *$ - Significant at $1 \%$ 
Table.5 Top three desirable hybrids with respect to sca effects for 10 characters in okra

\begin{tabular}{|c|c|c|c|c|c|}
\hline \multirow[t]{2}{*}{ Characters } & \multirow[t]{2}{*}{ Crosses } & \multirow[t]{2}{*}{ sca effect } & \multirow[t]{2}{*}{ Mean } & \multicolumn{2}{|c|}{ GCA study of } \\
\hline & & & & Female & Male \\
\hline \multirow[t]{3}{*}{ Days to $50 \%$ flowering } & DBh-55 x Arka Anamika & $-2.167 * *$ & 59.67 & low & high \\
\hline & DBh-43 x Arka Abhay & $-2.000 * *$ & 60.67 & low & high \\
\hline & Arka Abhay x DBh-37 & $-1.682 * *$ & 60.33 & high & low \\
\hline \multirow[t]{3}{*}{ Plant height $(\mathrm{cm})$} & DBh-43 x DBh-39 & $9.000 * *$ & 66.33 & high & high \\
\hline & DBh-47 x DBh-37 & $8.267 * *$ & 72.13 & low & high \\
\hline & DBh-39 x DBh-47 & $6.970 * *$ & 91.33 & high & low \\
\hline \multirow{3}{*}{$\begin{array}{l}\text { Number of branches per } \\
\text { plant }\end{array}$} & DBh-30 x Arka Abhay & $0.667 * *$ & 3.00 & low & high \\
\hline & Arka Abhay x DBh-43 & $0.661 * *$ & 4.00 & high & low \\
\hline & Arka Abhay x DBh-30 & $0.578 * *$ & 4.33 & high & low \\
\hline \multirow[t]{3}{*}{ Inter nodal length $(\mathrm{cm})$} & DBh-47 x DBh-55 & $-0.700 * *$ & 4.30 & high & low \\
\hline & DBh-47 x DBh-43 & $-0.539 * *$ & 4.30 & high & low \\
\hline & DBh-55 x DBh-43 & $-0.420 *$ & 5.40 & low & low \\
\hline \multirow[t]{3}{*}{ Fruit length $(\mathrm{cm})$} & DBh-47 x Arka Anamika & $0.983 * *$ & 14.83 & low & high \\
\hline & DBh-47 x DBh-37 & $0.683 * *$ & 13.87 & low & low \\
\hline & DBh-30 x DBh-55 & $0.405 *$ & 14.57 & low & low \\
\hline \multirow[t]{3}{*}{ Fruit diameter $(\mathrm{cm})$} & DBh-47 x DBh-39 & $6.667 *$ & 1.83 & low & high \\
\hline & Arka Anamika x DBh-37 & $6.406 * *$ & 1.90 & low & low \\
\hline & DBh-30 x DBh-55 & $5.990 *$ & 1.90 & low & low \\
\hline \multirow[t]{3}{*}{ Number of fruits per plant } & Arka Anamika x DBh-43 & $3.073 * *$ & 20.67 & high & high \\
\hline & DBh-47 x DBh-30 & $2.500 * *$ & 10.67 & low & low \\
\hline & DBh-43 x DBh-39 & $2.333 * *$ & 12.00 & high & low \\
\hline \multirow[t]{3}{*}{ Average fruit weight (g) } & DBh-43 x Arka Anamika & $2.167 * *$ & 12.0 & low & high \\
\hline & DBh-47 x Arka Anamika & $2.000 * *$ & 11.0 & low & high \\
\hline & Arka Anamika x DBh-47 & $1.458 * *$ & 16.0 & high & low \\
\hline \multirow[t]{3}{*}{ Fruit yield per plant (g) } & Arka Anamika x DBh-47 & $52.853 * *$ & 255.29 & high & low \\
\hline & DBh-43 x Arka Anamika & $50.00 * *$ & 232.88 & high & high \\
\hline & DBh-39 x DBh-43 & $39.858 * *$ & 200.44 & low & high \\
\hline \multirow{3}{*}{$\begin{array}{l}\text { Fruit yield per hectare } \\
\text { (T/ha) }\end{array}$} & Arka Anamika x DBh-43 & $2.627 * *$ & 15.84 & high & high \\
\hline & DBh-47 x Arka Anamika & $2.032 * *$ & 9.49 & low & high \\
\hline & DBh-47 x DBh-30 & $1.607 * *$ & 7.53 & low & low \\
\hline
\end{tabular}

The parents viz., Arka Anamika, Arka Abhay and DBh-43 identified as good general combining ability for fruit yield can be further tried with new parental combination for realizing higher magnitude of heterosis. Among hybrids, Arka Anamika x DBh-43 had higher sca score and is a good combiner for seven traits like plant height, number of branches, fruit length, number of fruits per plant, fruit weight, fruit yield per plant and fruit yield per hectare. Arka Anamika x DBh-37 and DBh-30 $x$ DBh-55 crosses have higher scores followed by crosses DBh-43 x Arka Anamika, DBh-47 x DBh-37 and DBh-47 x DBh-39 (Table 3 and 4).

For all the characters studied except days to 50 per cent flowering, plant height, number of branches and fruit yield per plant the low x low combination of gca status were present in heterotic cross combinations indicating predominance of non-additive gene action and very less of additive gene action in these crosses. The study of high yielding top three hybrids revealed that fruit yield was high in crosses involving lines exhibiting majority of high $\mathrm{x}$ low and low x high gca effects. The study on sca effects revealed that the performance of the hybrids for all the traits was higher when the parents were of high $\mathrm{x}$ high, high $\mathrm{x}$ low and low $\mathrm{x}$ low gca status, which indicates presence of additive and non-additive gene action. The high $\mathrm{x}$ low gca parental combinations resulted in the higher frequency of significant heterosis for different traits in the present study. The sca effects for fruit yield per hectare were positive and significant in the hybrids of which the highest sca effects with positive significance was in hybrid Arka Anamika x DBh-43. It is suggested to evaluate the hybrids Arka Anamika x DBh-43, DBh-37 x 
Arka Abhay and Arka Anamika x DBh-47 over locations and seasons to confirm their potentiality for exploitation of heterosis and their use in commercial cultivation. The hybrid combination exhibiting highest per se performance also manifested high sca effects, justifying the existence of high degree of dominance and additive gene action (Table 5).

Arka Anmika had higher gca scores and is a good combiner for days to 50 per cent flowering, fruit length, number of fruits per plant, fruit weight, fruit yield per plant and fruit yield per hectare, followed by Arka Abhay and DBh-43. The study on sca effects revealed that the performance of the hybrids for all the traits was higher when the parents were of high $\mathrm{x}$ high, high $\mathrm{x}$ low and low $\mathrm{x}$ low gca status, which indicates presence of additive and nonadditive gene action. It is suggested to evaluate the hybrids Arka Anamika x DBh-43, DBh-37 x Arka Abhay and Arka Anamika x DBh-47 over locations and seasons to confirm their potentiality for exploitation of heterosis and their use in commercial cultivation.

\section{References}

Ahmed, N., M.A. Hakim and G.H. Zargar, Combining ability studies in okra (Abelmoschus esculentus (L.) Moench.). Veg. Sci., 24, 1997, 95-98.

Chaudhary, D.R., Jagmohankumar, Vidyasagar and S.K. Sharma, Line x Tester analysis of combining ability in okra (Abelmoschus esculentus (L.) Moench.). South Indian Hort., 39 (6), 1991, 337340.

Dahake, K.D., and N.D. Bangar, Combining ability analysis in okra. J. Maharashtra Agric. Univ., 31 (1), 2006, 039-041.
Dhankar, B.S., and S.K. Dhankar, Heterosis and combining ability studies for some economic characters in okra. Haryana $J$. Hort. Sci., 30 (3\&4), 2002, 230-232.

Griffing, B., Concept of general and specific combining ability in relation to diallel crossing system. Australian J. Biol. Sci., 9, 1956, 463-493.

Poshiya, V.K., and P.T. Shukla, Combining ability in okra (Abelmoschus esculentus (L.) Moench.). Gujarath Agric. Univ. J., 12 (12), 1986, 25-28.

Sanjay Singh, B. Singh and A.K. Pal, Line x Tester analysis of combining ability in okra. Indian J. Hort. December, 2006, pp. 397-401.

Senthil Kumar, P., P. Sriram, and P. Karuppiah, Studies on combining ability in okra (Abelmoschus esculentus (L.) Moench). Indian J. Hort. 63 (2), 2006, 182-184.

Sharma, B.R., and Y.P. Mahajan, Line and tester analysis of combining ability and heterosis for some economic characters in okra. Scientia Hort., 9, 1978, 111-118.

Shukla, A.K., N.C. Gautam, A.K. Tiwari and A. K. Chaturvedi, Heterosis and combining ability in okra (Abelmoschus esculentus (L.) Moench.). Veg. Sci., 16, 1989, 191196.

Sivagamasundhari, S., I. Irulappan, R. Arumugam, and S. Jayasankar, combining ability in bhendi (Abelmoschus esculentus (L.) Moench). South Indian Hort., 40, 1992, 21-27.

Vijay, O.P., and M.S. Manohar, Heterosis and Combining ability in okra (Abelmoschus esculentus (L.) Moench.). Indian J. Hort., 43, 1986, 133-139.

Wankhade, R.V., P.B. Kale, V.N. Dod, Combining ability in okra. Indian $J$. Hort., 19 (2), 1991, 121-124.

\section{How to cite this article:}

Amaranatha Reddy, M. and Sridevi, O. 2018. Combining Ability for Yield and Yield Components through Diallel Analysis in Okra [Abelmoschus esculentus (L.) Moench]. Int.J.Curr.Microbiol.App.Sci. 7(03): 1023-1029. doi: https://doi.org/10.20546/ijcmas.2018.703.122 